\title{
Phase transitions in porous materials: influence of physico-chemical heterogeneities
}

\author{
J. Puibasset ${ }^{1}$ \\ ${ }^{1}$ Centre de Recherche sur la Matière Divisée, CNRS Université d'Orléans, 1 bis rue de la Férollerie, 45071 Orléans cedex \\ 2, France
}

\section{Introduction}

Essentially all surfaces adsorb fluids due to favourable interactions [1, 2]. For strong enough interactions, chemical bonds may be broken in the adsorbed molecules and/or established between the adsorbent and the adsorbate (chemisorption). For weak interactions, the adsorption process occurs reversibly without breaking any molecule (physisorption). Despite weak, the fluid/substrate interactions induce observable modifications of the fluid properties in the vicinity of the surface. Moreover, collective effects may be induced when confining fluids in porous materials, because the local curvature associated to porosity enhances fluid/substrate interactions. The measured adsorption isotherms (giving the amount of fluid adsorbed versus fluid pressure) thus provide a powerful experimental tool for porous materials characterization [3, 4]. An important feature of such isotherms is their possible irreversibility between adsorption and desorption (existence of a hysteresis, to be discussed later).

Focusing on heterogeneous surfaces, we show how surface chemistry and geometry influence the adsorption properties of a condensable fluid and hence its liquid/vapor coexistence diagram. In most cases, molecular simulation, taking into account the fluid/substrate interactions at atomistic scale, has proven to be a powerful tool to understand adsorbed fluid properties in the vicinity of a surface. In particular for studying the effect of surface chemistry or corrugation, local curvature, pore morphology, or any other nanometer-scale property of the surface

We show that, increasing the surface heterogeneities has drastic effects on coexistence diagram. Not only a shift of the critical point (i.e. quantitative changes), but also the appearance of many metastable states that introduce qualitative changes [5, 6]. A multi-scale approach has been used to explore this issue, showing the importance of the interdependence between the various heterogeneities for the adsorption and desorption mechanisms, the observed hysteresis, and the distribution of the underlying metastable states. Such approach, including both an atomistic and a large-scale description (from interatomic forces to large scale domain network) is expected to offer a powerful tool for experimental data analysis.

\section{Multiscale approach}

In this section we introduce briefly the multi-scale molecular simulation approach. Molecular simulation of realistic systems is difficult because of computational limitations: pores larger than $10 \mathrm{~nm}$ are difficult to handle, and in most experimental situations the adsorbent exhibits strong heterogeneities. As introduced by Everett [7-9] a porous material can be seen as a collection of domains corresponding to elemental subdivision of the porous network (elemental cavities). The most widely used approach consists in considering that the domains are independent (see Fig. 1).
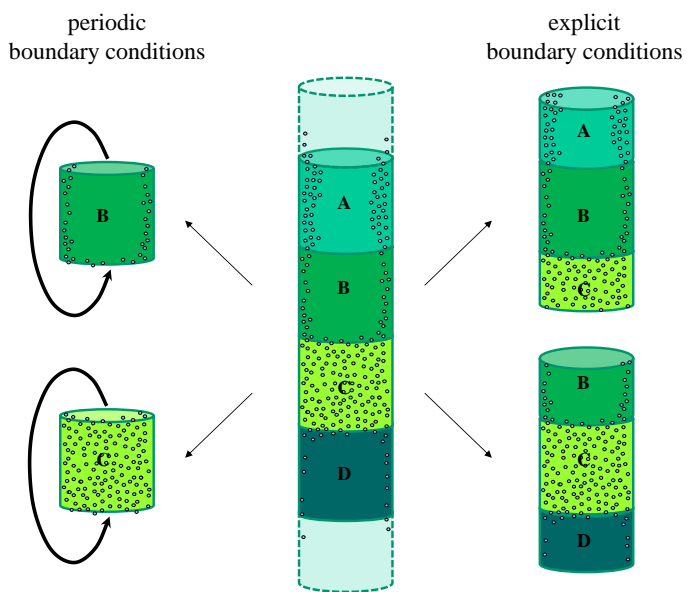

Figure 1. Schematic representation of the heterogeneous pore (centre). Left: all domains are treated independently, with periodic boundary conditions. Right: multiscale approach: all domains are treated taking into account the neighbours with explicit boundary conditions.

The global thermodynamic properties are thus obtained as an average over the domain distribution. When typical domains are of nanometric scale, molecular simulation is able to treat each of them independently and thus provides a powerful analysis of fluid adsorption after 
averaging over the appropriate distribution of domains. This approach leads to an extremely accurate description of the low pressure region of the adsorption isotherm, where the hypothesis of independence between domains is well satisfied.

The hysteresis region is however not well described by this approach because the disorder associated to the complex porous network plays an important role. This has been shown by using (simple) network models [1012] or more sophisticated coarse grained approaches [1316]. What do we learn from these models? In the hysteresis region, the fluid adsorbed in a given domain is not uniquely defined and depends on the fluid state in the neighbouring domains (existence of metastable states). The rules defining this dependence are generally deduced from a classical thermodynamic analysis of fluid adsorption in pores. However, significant improvement can be achieved if one uses molecular simulation to extract them, with appropriate explicit boundary conditions to take into account the presence of neighbouring domains $[17,18]$.

\section{Molecular model}

We focus on simple fluid adsorption in the frequently encountered silica-based nanoporous materials like MCM-41 or oxidized porous silica. These materials exhibit parallel and non-interconnected tubular pores with a high aspect ratio (few nanometers in diameter and few microns of length) [19-21]. Such materials are of greatest fundamental interest since they exhibit unexpected hysteresis shapes [22-24]. Due to fabrication procedure, one expects physico-chemical variations at nanometer scale along the pore axis [25-29] which essentially result in a modulation of the affinity of the adsorbed fluid (argon in our case) with the wall. We have in particular shown that pore size variations actually predominantly result in variations of fluid/wall interactions, with moderate "purely geometric" effects [30-33]. As a simplification, the corresponding heterogeneities are characterized by a single parameter which varies along pore, and measures the intensity of the fluid/wall interaction.

The $3 \mathrm{~nm}$ in diameter pore is drilled in an initial substrate made of pure silica of density close to the expected one (mesoporous silica walls are expected to be made of amorphous silica). The fluid/fluid and fluid/substrate interactions are modeled by the (6-12) Lennard-Jones intermolecular potential cut and shifted at $2.5 \sigma$. The parameters for argon are $\sigma_{\mathrm{Ar}-\mathrm{Ar}}=0.3405 \mathrm{~nm}$ and $\varepsilon_{\mathrm{Ar}-\mathrm{Ar}} / k=120 \mathrm{~K}$ where $k$ is Boltzmann constant, and the parameters for argon/silica interactions are $\varepsilon_{\mathrm{Ar}-\mathrm{O}}^{0} / k=100 \mathrm{~K}$ and $\sigma_{\mathrm{Ar}-\mathrm{O}}=0.333 \mathrm{~nm}$ (fluid/wall interactions are mainly with $\mathrm{O}$ species). The physicochemical heterogeneities in the porous substrate are approximated by a modulation over the porous substrate of the fluid/wall interaction intensity $\varepsilon_{\mathrm{Ar}-\mathrm{O}}$ around its typical value $\varepsilon_{\mathrm{Ar}-\mathrm{O}}^{0}$, within \pm 30 percent. The heterogeneity parameter $h=\varepsilon_{\mathrm{Ar}-\mathrm{O}} / \varepsilon_{\mathrm{Ar}-\mathrm{O}}^{0}$ is supposed to be constant within each domain, and to take uncorrelated values between two adjacent domains. Its value within a given pore is supposed to follow a Gaussian distribution.

The simulations are performed using the grand canonical Monte Carlo algorithm [34, 35]. The chemical potential of argon is imposed and related to the gas pressure in the reservoir using the ideal gas relation since it is a good approximation at the temperature at which the simulations are performed $\left(T^{*}=T k / \varepsilon_{\mathrm{Ar}-\mathrm{Ar}}=0.6\right.$, which corresponds approximately to $70 \mathrm{~K})$. The adsorption/desorption isotherms are given as a function of the reduced chemical potential $\mu^{*}=\mu / \varepsilon_{\mathrm{Ar}-\mathrm{Ar}}$. Long simulations are performed in order to reduce the uncertainties down to $7 \%\left(10^{8}\right.$ Monte Carlo trials per argon where required).

\section{Results}

The adsorption/desorption isotherms obtained within the independent domain theory and the multiscale approach are given in Fig. 2. The equilibrium isotherm, which corresponds to the absolute minimum of the grand potential, is also given (dotted line through the hysteresis). As can be seen, the isotherms present a large hysteresis, with smoothly increasing (adsorption) or decreasing (desorption) branches (no sharp transition). The second noticeable point is that the equilibrium curve is always between the adsorption and desorption branches.

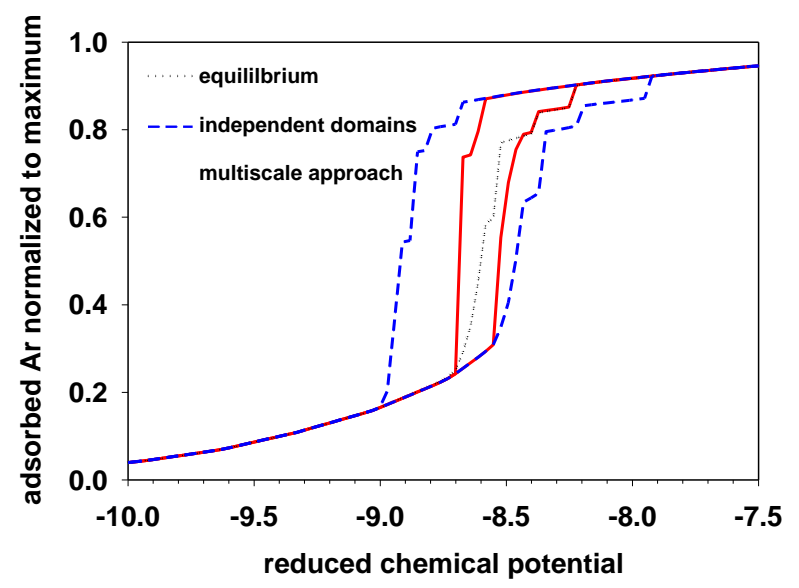

Figure 2. Adsorption/desorption isotherms for Argon in porous silica ( $3 \mathrm{~nm}$ in diameter) obtained by Monte Carlo simulation.

Dotted line: equilibrium; dashed (blue) lines: independent domains; solid (red) line: interdependent domains (multiscale approach).

As can be seen, the adsorption/desorption curves present a hysteresis which is significantly smaller when the interdependence between pores is taken into account. The difference comes from the fact that adsorption starts in the most attractive domains, creating a meniscus. If the interdependence between domains is taken into account, the meniscus is allowed to propagate to neighbouring 
domains. Note that the presence of a meniscus does not necessarily means that adsorption occurs at equilibrium, since the meniscus propagation may be delayed by the less attractive regions of the pore. Upon desorption, similar mechanism of meniscus propagation is exhibited (recession from the open pore end). The main difference when taking into account interdependence between domains is that the adsorption branch meets the equilibrium curve before the highest closure point, and the desorption branch meets the equilibrium curve at the lowest closure point.

\section{Discussion and conclusion}

The simulation results obtained in the multiscale approach show that, despite the presence of menisci during adsorption/desorption, the system is not at equilibrium. It is however frequently supposed that at least one of the adsorption or desorption branches occurs at equilibrium, which can then be used to evaluate the pore size distribution. This study shows that none of the branches is at equilibrium, and hence the standard methods to obtain pore size distributions are poorly justified. Collaboration with experimentalists is underway to use this multiscale approach for improving experimental data analysis.

\section{References}

1. J.H. Cushman, The Physics of Fluids in Hierarchical Porous Media: Angstroms to Miles, Kluwer Academic Publishers, London, 1997

2. Y. Guéguen and V. Palciauskas, Introduction to the physics of rock, University Press, Princeton, 1994

3. S.J. Gregg and K.S.W. Sing, Adsorption, Surface Area and Porosimetry, Academic Press, New York, 1982

4. F. Rouquerol, J. Rouquerol, K.S.W. Sing, Adsorption by Powders and Porous Solids, Academic Press, London, 1999

5. J. Puibasset, J. Chem. Phys. 125074707 (2006)

6. J. Puibasset, J. Chem. Phys. 129024705 (2008)

7. D.H. Everett and W.I. Whitton, Trans. Faraday Soc. 48749 (1952)

8. D.H. Everett and F.W. Smith, Trans. Faraday Soc. 50 187 (1954)

9. D.H. Everett, Trans. Faraday Soc. 501077 (1954)

10. G. Mason, J. Colloid Interface Sci. 8836 (1982)

11. G. Mason, Proc. R. Soc. London A 39047 (1983)

12. M.R. Swift, E. Cheng, M.W. Cole, J.R. Banavar, Phys. Rev. B 483124 (1993)

13. E. Kierlik, M.L. Rosinberg, G. Tarjus, P. Viot, Phys. Chem. Chem. Phys. 31201 (2001)

14. E. Kierlik, P.A. Monson, M.L. Rosinberg, L. Sarkisov, G. Tarjus, Phys. Rev. Lett. 87055701 (2001)

15. F. Detcheverry, E. Kierlik, M.L. Rosinberg, G. Tarjus, Phys. Rev. E 68061504 (2003)

16. F. Detcheverry, E. Kierlik, M.L. Rosinberg, G. Tarjus, Phys. Rev. E 73041511 (2006)

17. J. Puibasset, J. Chem. Phys. 127154701 (2007)
18. J. Puibasset, Langmuir 25903 (2009)

19. J.S. Beck, J.C. Vartuli, W.J. Roth, M.E. Leonowicz, C.T. Kresge, K.D. Schmitt, C.T.-W. Chu, D.H. Olson, E.W. Sheppard, S.B. McCullen et al., J. Am. Chem. Soc. 11410834 (1992)

20. C.T. Kresge, M.E. Leonowicz, W.J. Roth, J.C. Vartuli, J.S. Beck, Nature 359710 (1992)

21. A. Uhlir, Bell Syst. Tech. J. 35333 (1956)

22. B. Coasne, A. Grosman, C. Ortega, M. Simon, Phys. Rev. Lett. 88256102 (2002)

23. A. Grosman and C. Ortega, Langmuir 2110515 (2005)

24. A. Grosman and C. Ortega, Langmuir 243977 (2008)

25. M.W. Maddox, J.P. Olivier, K.E. Gubbins, Langmuir 131737 (1997)

26. K.J. Edler, P.A. Reynolds, J.W. White, J. Phys. Chem. B 1023676 (1998)

27. A. Berenguer-Murcia, J. Garcia-Martinez, D. CazorlaAmoros, A. Martinez-Alonso, J.M.D. Tascon, A. Linares-Solano, in Studies in Surface Science and Catalysis, F. Rodriguez Reinoso, B. McEnaney, J. Rouquerol, K. Unger Eds., Elsevier Science, Amsterdam, 2002

28. V.B. Fenelonov, A.Y. Derevyankin, S.D. Kirik, L.A. Solovyov, A.N. Shmakov, J.-L. Bonardet, A. Gedeon, V.N. Romannikov, Micropor. Mesopor. Mater. 44-45 $33(2001)$

29. C.G. Sonwane, C.W. Jones, P.J. Ludovice, J. Phys. Chem. B 10923395 (2005)

30. J. Puibasset, J. Phys. Chem. B 1094700 (2005)

31. J. Puibasset, J. Chem. Phys. 122134710 (2005)

32. J. Puibasset, J. Phys. Chem. B 109480 (2005)

33. J. Puibasset, J. Phys. Chem. B 1098185 (2005)

34.D. Nicholson and N.G. Parsonage, Computer Simulation and the Statistical Mechanics of Adsorption, Academic Press, London, 1982

35. M.P. Allen and D.J. Tildesley, Computer Simulation of Liquids, Clarendon Press, Oxford, 1987 